\title{
Role of Glutamine Aminotransferase in Glutamine Catabolism by Saccharomyces cerevisiae under Microaerophilic Conditions
}

\author{
By MARIO SOBERÓN, JESÚS OLAMENDI, LAURA RODRÍGUEZ AND \\ ALICIA GONZÁLEZ* \\ Departamento de Biología Molecular de Plantas, Centro de Investigacion sobre Fijación de \\ Nitrógeno, UNAM, Apartado Postal 565-A, Cuernavaca, Morelos, Mexico
}

(Received 31 May 1989; accepted 6 June 1989)

The involvement of glutamine aminotransferase activity in glutamine catabolism by Saccharomyces cerevisiae under microaerophilic conditions was studied. We were able to show that there are at least two different glutamine aminotransferase activities that are differentiated genetically, by their substrate specificity (pyruvate and glyoxylate dependence), and their different modes of regulation. The pyruvate-dependent glutamine aminotransferase activity plays a major role in glutamine catabolism under microaerophilic conditions since the wild-type strain S288C showed a 10-fold higher activity in static cultures than in agitated ones. The same strain also had 3-fold higher glutaminase B activity in agitated cultures than in static ones. Pyruvate-dependent glutamine aminotransferase activity is not regulated directly by $\mathrm{O}_{2}$ itself since a $\rho^{-}$strain showed a high activity regardless of the extent of aeration of cultures. Finally, we were able to isolate a mutant, strain $\mathrm{CN} 20$, derived from the $\rho^{-}$strain and unable to utilize glutamine as the sole nitrogen source, which was severely affected in pyruvate-dependent but not in glyoxylate-dependent aminotransferase activity.

\section{INTRODUCTION}

Glutamine plays a key role in nitrogen metabolism; it supplies nitrogen required for the biosynthesis of a variety of nitrogenous metabolic intermediates in most organisms, including Saccharomyces cerevisiae. Glutamine has also been proposed to be the co-repressor of nitrogen catabolite repression in yeast (Dubois et al., 1977; Legrain et al., 1982). Due to the dual role that glutamine plays, its biosynthesis and degradation may be $a$ priori precisely regulated in order to maintain an intracellular concentration that will support biosynthesis without resulting in repression. Our previous work concerned the role of glutaminase (EC 3.5.1.2) in glutamine assimilation in S. cerevisiae (Soberón \& González, 1987a). We found that there are at least two glutaminases (A and B) in this yeast and that these have different $\mathrm{pH}$ optima. Glutaminase $\mathrm{A}$ is membrane bound whilst glutaminase B is cytoplasmic. Also, glutaminase B is negatively modulated by organic acids such as pyruvate, glyoxylate and 2-oxoglutarate (Soberón \& González, 1987a). Additionally, the $\omega$-amidase pathway constitutes an alternative route for glutamine catabolism, and $\omega$-amidase activity has been demonstrated in $S$. cerevisiae (Cooper \& Meister, 1977). In this pathway, glutamine is transaminated to yield different amino acids and 2-oxoglutaramate, through the catalytic activity of glutamine aminotransferase; subsequently, 2 -oxoglutaramate is hydrolysed by $\omega$-amidase to 2-oxoglutarate and ammonium. The existence and function of the $\omega$-amidase pathway in mammalian systems is well-documented (Cooper $\&$ Meister, 1981). We have studied the $\omega$-amidase pathway in $S$. cerevisiae, in which the highest glutamine aminotransferase activity is found during growth in the presence of glutamate or glutamine, with or without ammonium (Soberón \& González, 1987 b). This activity can be detected using various 2-oxoacids (Soberón \& González, 1987b). Our results suggest that the

Abbreviation: MM, Minimal medium. 
$\omega$-amidase pathway and the alternative pathway via glutaminase $B$ are inversely regulated. That is, under microaerophilic conditions in which pyruvate accumulates, glutaminase B is inhibited and glutamine is degraded by the $\omega$-amidase pathway (Soberón \& González, 1987b); under aerobic conditions, where the accumulation of pyruvate is less, glutamine is catabolized by glutaminase B. Our model predicts that a respiratory-deficient mutant strain should degrade glutamine through the $\omega$-amidase pathway regardless of the state of aeration of the culture.

In this paper we report the isolation of a mutant defective in glutamine aminotransferase which is unable to grow on glutamine as sole nitrogen source. The fact that this mutant was derived from a respiratory-deficient $\left(\rho^{-}\right)$strain indicates that the $\omega$-amidase pathway is the main catabolic pathway used to degrade glutamine under microaerophilic conditions in which anaerobic metabolism is favoured.

\section{METHODS}


(MATa; leu2-3; leu2-112; his3; canl) were obtained from the Cold Spring Harbor Laboratory, NY, USA.

Growth conditions. Strains were routinely grown in minimal media (MM) containing salts, trace elements and vitamins following the formula of Difco Yeast Nitrogen Base. Glucose $(2 \%, \mathrm{w} / \mathrm{v})$ was used as carbon source and $40 \mathrm{mM}$ - $\left(\mathrm{NH}_{4}\right)_{2} \mathrm{SO}_{4}$ (i.e. $80 \mathrm{~mm}$ nitrogen), or $6.8 \mathrm{~mm}$-glutamine, as nitrogen source. Cells were incubated at $30^{\circ} \mathrm{C}$ with orbital shaking $\left(175\right.$ r.p.m.). Static cultures were incubated at $30^{\circ} \mathrm{C}$ without shaking. Growth was measured by determining the total protein content of cultures. Samples $(20 \mathrm{ml})$ were filtered through $0.22 \mu \mathrm{m}$ Millipore filters and cells were washed with distilled water. Resuspended samples were then precipitated with $2 \mathrm{ml} 5 \%$ trichloroacetic acid and centrifuged for $5 \mathrm{~min}$ at 2000 r.p.m. The pellets were suspended in $1.0 \mathrm{M}-\mathrm{NaOH}$ and samples $(0.1-0.2 \mathrm{ml})$ used for protein determination. This was done by the Lowry method using BSA as a standard.

Mutagenesis and mutant selection. To obtain mutants unable to use glutamine as nitrogen source, strain DS400/A12 was mutagenized by treatment with ethylmethane sulphonate (EMS), following the method of Fink (1970). After mutagenesis, the cells were allowed to grow for $24 \mathrm{~h}$ on $\mathrm{MM}$ plus $0.01 \%$ each of alanine, glycine, tyrosine and tryptophan with $40 \mathrm{mM}-\left(\mathrm{NH}_{4}\right)_{2} \mathrm{SO}_{4}$ as nitrogen source. These amino acids were added since their 2-oxoacids are substrates of glutamine aminotransferase, and an alteration in this activity could result in an auxotrophic requirement for these compounds. Cells that could not use glutamine as a nitrogen source were then selectively enriched using the antibiotic $N$-glycosyl-polyfungin (Polaina \& Conde, 1981). Cells were spread on the amino-acid-supplemented medium described above and incubated at $30^{\circ} \mathrm{C}$. Colonies which appeared after 3-5 d were replica-plated to MM plates containing $40 \mathrm{mM}-\left(\mathrm{NH}_{4}\right)_{2} \mathrm{SO}_{4}$ or $7.8 \mathrm{mM}$-glutamine as nitrogen source plus the above-mentioned amino acids. Cells which failed to grow on the glutamine plates were purified and further characterized.

Determination of glutaminase $B$ activity. Soluble extracts were prepared by grinding whole cells in $50 \mathrm{~mm}$ Tris/ $\mathrm{HCl}$ buffer, $\mathrm{pH} 8 \cdot 1$, using a Braun homogenizer and glass beads. The extracts were centrifuged $(20 \mathrm{~min}$, $9000 \mathrm{~g}, 4^{\circ} \mathrm{C}$ ) and the supernatant was used as the source of enzyme.

The activity was assayed in reaction mixtures $(0.5 \mathrm{ml})$ containing $50 \mathrm{mM}-\mathrm{Tris} / \mathrm{HCl}, \mathrm{pH} 8.1,6 \mathrm{~mm}-$ [U-14 ${ }^{14}$ ]glutamine $\left[0.045 \mu \mathrm{Ci} \mathrm{nmol}^{-1}\left(166.5 \mathrm{~Bq} \mathrm{nmol}^{-1}\right)\right]$ and $0.1 \mathrm{ml}$ of cell extract. The reaction mixture was incubated at $37^{\circ} \mathrm{C}$ for $20 \mathrm{~min}$ or $40 \mathrm{~min}$ and then the reaction stopped by adding $1.5 \mathrm{ml}$ cold $50 \mathrm{mM}$-Tris $/ \mathrm{HCl}$ buffer. This mixture was passed through a Dowex $1 \times 8-400$ column and washed with $20 \mathrm{ml}$ distilled water. $\left[\mathrm{U}-{ }^{14} \mathrm{C}\right]$ Glutamate was eluted from the column with $8 \mathrm{ml} 2 \mathrm{M}$-acetic acid and a sample $(0 \cdot 1 \mathrm{ml})$ was used to determine $\left[\mathrm{U}-{ }^{14} \mathrm{C}\right] \mathrm{glutamate}$ by liquid scintillation counting. Activity is expressed as nmol glutamate produced $\min ^{-1}$ (mg protein) ${ }^{-1}$.

Determination of glutamine aminotransferase activity. This was done following the method previously described (Soberón \& González, 1987a). Activity is expressed as nmol 2-oxoglutaramate produced $\mathrm{min}^{-1}$ (mg protein) ${ }^{-1}$.

Determination of glutamine and glutamate pools. Cells were harvested by centrifugation (10 min, 7000 r.p.m.) and immediately suspended in $80 \%(\mathrm{v} / \mathrm{v})$ ethanol and heated in boiling water for $10 \mathrm{~min}$. Cells were then broken using a Braun cell disrupter and debris was removed by filtration through $0.22 \mu \mathrm{m}$ Millipore filters. The filtrates containing the free amino acids were lyophilized and then resuspended in citrate $(0.053 \mathrm{M}) \mathrm{LiOH}(0.3 \mathrm{M})$ buffer (pH 2.88). The amino acids were separated with an Aminco Amino Acid Analyzer and quantified in an Aminco ratio fluorimeter after coupling with $o$-phthaldehyde.

Chemicals. All amino acids, EMS and bovine serum albumin were obtained from Sigma.

\section{RESULTS AND DISCUSSION}

Glutaminase $B$ and glutamine aminotransferase activities in the $\rho^{-}$strain

We have shown previously that glutaminase B activity is the major glutamine catabolic pathway when $S$. cerevisiae is cultured in aerobic conditions (Soberón \& González, 1987a), but 
Table 1. Glutaminase B and glutamine aminotransferase activities in extracts of strains S288C, DS400/A12 and CN20 grown statically or with agitation

Representative results of three experiments with a variation less than $15 \%$ are shown.

\begin{tabular}{|c|c|c|c|c|c|c|}
\hline \multirow[b]{2}{*}{ Enzyme. } & \multicolumn{3}{|c|}{ Agitated* } & \multicolumn{3}{|c|}{ Static $\dagger$} \\
\hline & \multirow{2}{*}{$\begin{array}{c}\text { Glutaminase } \\
\text { B }\end{array}$} & \multicolumn{2}{|c|}{ Glutamine aminotransferase } & \multirow{2}{*}{$\begin{array}{c}\text { Glutaminase } \\
\text { B }\end{array}$} & \multicolumn{2}{|c|}{ Glutamine aminotransferase } \\
\hline Strain & & $\begin{array}{l}\text { Pyruvate } \\
\text { dependent }\end{array}$ & $\begin{array}{l}\text { Glyoxylate- } \\
\text { dependent }\end{array}$ & & $\begin{array}{l}\text { Pyruvate- } \\
\text { dependent }\end{array}$ & $\begin{array}{l}\text { Glyoxylate- } \\
\text { dependent }\end{array}$ \\
\hline $\mathrm{S} 288 \mathrm{C}\left(\rho^{+}\right)$ & $22 \cdot 30$ & 1.07 & $4 \cdot 86$ & $6 \cdot 70$ & $10 \cdot 13$ & 3.06 \\
\hline DS400/A12 $\left(\rho^{-}\right)$ & $0 \cdot 291$ & $9 \cdot 28$ & 3.55 & ND & $5 \cdot 70$ & $10 \cdot 63$ \\
\hline $\mathrm{CN} 20\left(\rho^{-}\right)$ & 3.35 & $0 \cdot 18$ & $6 \cdot 00$ & $1 \cdot 30$ & 0.50 & 12.90 \\
\hline
\end{tabular}

ND, Not detected.

* 240 r.p.m.: volume of medium/flask capacity $=1 / 6$.

$\dagger$ Volume of medium/flask capacity $=1 / 2$.

not under microaerophilic culture conditions (Soberón \& González, 1987b). We therefore measured glutaminase B and glutamine aminotransferase activities in a wild-type strain (S288C) and in a $\rho^{-}$strain (DS400/A12) cultured with glutamine as the sole nitrogen source under different conditions of aeration (Table 1 ).

Strain S288C had at least 3-fold lower glutaminase B activity in nonagitated cultures (Table 1) as was shown previously (Soberón \& González, 1987a). Further, pyruvate-dependent glutamine aminotransferase activity was 10 -fold higher in nonagitated cultures as compared to wellaerated cultures, whilst glyoxylate-dependent glutamine aminotransferase activity varied little with aeration conditions. These results indicate that pyruvate-dependent glutamine aminotransferase activity could play a major role in glutamine catabolism under microaerophilic culture conditions.

Strain DS400/A12 showed very low levels of glutaminase B activity regardless of aeration conditions (Table 1). This was expected since we previously proposed that glutaminase B activity is negatively regulated by some oxoacids, including pyruvate which accumulates under microaerophilic conditions (Soberón \& González, 1987a); thus a $\rho^{-}$strain would accumulate pyruvate regardless of the aeration conditions. In strain DS400/A12 pyruvate-dependent glutamine aminotransferase activity was similar in both well-aerated and nonagitated cultures. These results suggest that in the $\rho^{-}$strain glutamine is catabolized by the pyruvate-dependent glutamine aminotransferase under aerobic and microaerophilic conditions, and that this activity is regulated by the metabolic status of the cell rather than directly by the $\mathrm{O}_{2}$ tension.

\section{Isolation and characterization of a mutant impaired in pyruvate-dependent glutamine aminotransferase activity}

Pyruvate-dependent glutamine aminotransferase activity appeared to play a major role in glutamine catabolism in the DS400/A12 $\left(\rho^{-}\right)$strain regardless of culture conditions (see above). We therefore mutagenized this $\rho^{-}$strain to look for mutants unable to grow on glutamine as sole nitrogen source. We isolated a mutant (CN20) which has significantly more-impaired growth on glutamine than on ammonium as sole nitrogen source (Fig. 1). Strain CN20 was mated with strain $\mathrm{AH} 22$, and diploids obtained were able to grow on glutamine showing that the mutation present was recessive (data not shown). Moreover, when the diploids were sporulated, the spores were non-viable. This phenotype could be due to a high intracellular glutamine content, since a mutant altered in glutaminase B shows a similar phenotype with respect to sporulation (Soberón \& González, 1987a).

We measured glutaminase B and glutamine aminotransferase (pyruvate- and glyoxylatedependent) activities in strain CN20 cultured on glutamine as sole nitrogen source. Table 1 shows that strain CN20 had a higher glutaminase B activity than DS400/A12 in both static and agitated cultures; nevertheless, these activities were significantly lower than those in the $\rho^{+}$ 


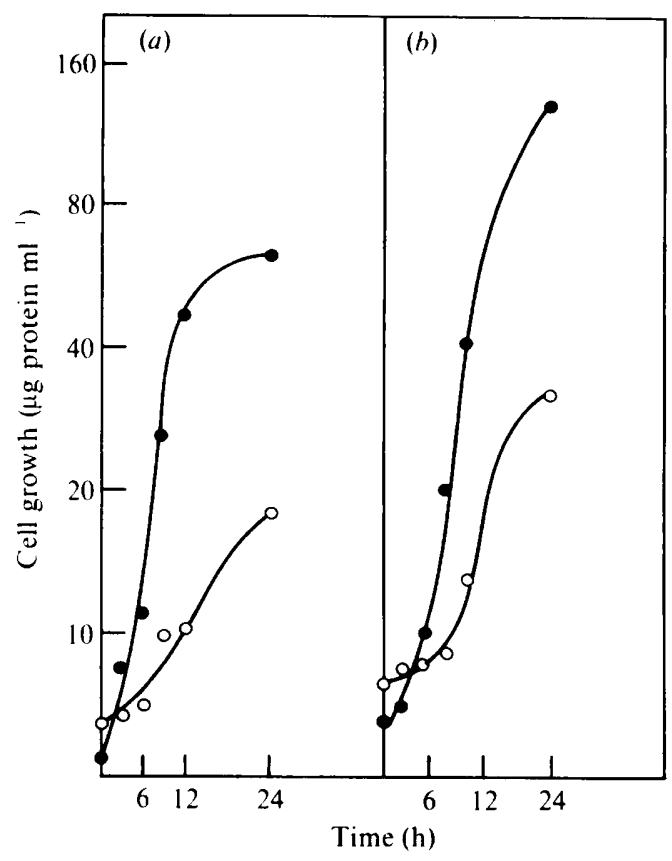

Fig. 1. Growth of strains DS400/A12 (O) and CN20 (O) on $0 \cdot 1 \%(\mathrm{w} / \mathrm{v})$ glutamine $(a)$ or $40 \mathrm{mM}-$ $(\mathrm{NHy})_{2} \mathrm{SO}_{4}(b)$. Methionine was added at a final concentration of $0.01 \%(\mathrm{w} / \mathrm{v})$ to complement auxotrophy. Growth was determined as total cell protein.

strain. Strain CN20 showed 5-fold lower and 25-fold lower pyruvate-dependent glutamine aminotransferase activity in agitated and static cultures respectively than its parent strain, but similar glyoxylate-dependent glutamine aminotransferase activities.

Since an impairment in glutamine catabolism should result in glutamine accumulation, we measured intracelullar glutamine and glutamate pools in the strains CN20 and DS400/A12 grown on glutamine. $\mathrm{CN} 20$ had 3-fold and 4-fold higher glutamine and glutamate pools than DS400/A12. [0.200 and $0.068 \mu \mathrm{mol}$ glutamine (mg protein) $)^{-1}$ and 0.300 and $0.068 \mu \mathrm{mol}$ glutamate (mg protein) ${ }^{-1}$ for strains CN20 and DS400/A12 respectively. Cells grown for $12 \mathrm{~h}$ in agitated cultures with $0 \cdot 1 \%(\mathrm{w} / \mathrm{v})$ glutamine as sole nitrogen source.] This implies that growth impairment of CN20 is not due to a glutamate requirement. Glutamate accumulation in CN20 could be explained by the presence of glutaminase A, which we have shown previously to be responsible for glutamate accumulation in a mutant strain that lacks glutaminase B (Soberón \& González, 1987a).

The results obtained suggest the existence of two different glutamine aminotransferase enzymes since the pyruvate- and the glyoxylate-dependent activities were regulated in different ways and the CN20 mutant strain was affected only in the pyruvate-dependent activity. Also, we have been able to show that the glyoxylate-dependent activity has at least two $\mathrm{pH}$ optima (data not shown). Further research is in progress to characterize the different glutamine aminotransferase activities with respect to substrate specificity and their role in glutamine catabolism. Nevertheless, the results presented suggest that the pyruvate-dependent glutamine aminotransferase activity is the major glutamine catabolic pathway in $S$. cerevisiae cultured under microaerophilic conditions.

The authors are grateful to Gloria Soberón for her critical review of the manuscript, to Concepción Hernández for typing the manuscript and to Herlinda Manrique for her secretarial assistance.

The work was supported in part by a grant from Fondo de Estudios de Investigaciones Ricardo J. Zevada. 


\section{REFERENCES}

COOPER, A. J. L. \& Meister, A. (1977). The glutamine transaminase $\omega$-amidase pathway. Critical Reviews in Biochemistry 4, 281-303.

CoOper, A. J. L. \& Meister, A. (1981). Comparative studies of glutamine transaminases from rat tissues. Comparative Biochemistry and Physiology 69, 137145.

Dubois, E., Vissers, S., Grenson, M. \& Wiame, J. M. (1977). Glutamine and ammonia in nitrogen catabolite repression of Saccharomyces cerevisiae. Biochemical and Biophysical Research Communications 75, 233-239.

FINK, G. R. (1970). The biochemical genetics of yeast. Methods in Enzymology 16, 58-78.

Legrain, C., Vissers, S., Dubois, E., Legrain, M. \&
WiAME, J. M. (1982). Regulation of glutamine synthesase from Saccharomyces cerevisiae by repression, inactivation and proteolysis. European Journal of Biochemistry 123, 611-616.

Polaina, J. A. \& Conde, J. (1981). Use of the polyene antibiotic $\mathrm{N}$-glycosyl-polifungin in counterselecting yeast mutants. Mutation Research 91, 111-114.

Soberón, M. \& González, A. (1987a). Physiological role of glutaminase activity in Saccharomyces cerevisiae. Journal of General Microbiology 133, 1-8.

Soberón, M. \& González, A. (1987b). Glutamine degradation through the $\omega$-amidase pathway in Saccharomyces cerevisiae. Journal of General Microbiology 133, 9-14. 